\title{
Vehicle velocity estimation based on uncertainty theory for vehicle impact accidents
}

\author{
Haocun Dong ${ }^{1}$, Jiaqi Yuan ${ }^{2}$, Zhongguo $\mathrm{Nie}^{3}$ \\ ${ }^{1}$ Automobile and Traffic College, Shenyang Ligong University, Shenyang 110159, China \\ ${ }^{2}$ Shenyang Longchang Pipeline Survey Center, Shenyang, 110031, China \\ ${ }^{3}$ Shenyang Jiashi Judicial Identification Office, Shenyang 110025, China \\ \{Haocun Dong, Jiaqi Yuan, Zhongguo Nie. Donghaocun@163.com
}

\begin{abstract}
Based on the uncertainty theory, a corresponding uncertainty analysis method for speed calculation model for the road traffic accidents of vehicle impact is set up.
\end{abstract}

\section{Introduction}

To some extent, the severity of traffic accidents depends on the impacting characteristics of velocity of vehicle impact [1]. Determining vehicle impact velocity is an important basis for identification of road traffic accidents, however, the actual accident velocity estimation will use some physical accident parameters, whether it is the accident scene measurement parameters such as vehicle braking distance, or the experience parameters such as wheel and road surface adhesion coefficient, which all contain uncertain information, that is, the accident parameter has uncertainty $[2,3,4]$, which inevitably leads to the uncertainty of estimation result of the traffic accident velocity. How to judge the random uncertainty of the accident velocity estimation result caused by the uncertainty of these parameters is a key problem [5].

The uncertainty of the parameters included in the vehicle impact traffic accidents is studied by the uncertainty theory, the range of the velocity before impact is deduced according to the uncertainty of these parameters, and the accuracy and confidence of the calculation results are analyzed to improve the reliability of the calculation results, and to make a scientific and impartial analysis of road traffic accidents [6,7].

\section{Brief description of the basic theory of uncertainty}

For a certain measurement parameter, because the real value is not an ideal concept of the concrete value, it is impossible to give the error size, but only the range of error value can be given, and use the uncertainty to describe the uncertainty of the parameters. Measurement of uncertainty is used to represent the dispersion of the measured value, which is an estimate of the true value of the measured value in a certain value range. Measurement of uncertainty usually contains several components, and all the uncertainty components are characterized by standard deviation. The categories of uncertainty can be 
divided into standard uncertainty, synthetic standard uncertainty and extension uncertainty (also known as expanded uncertainty). The uncertainty characterized by standard deviation is the standard uncertainty. The uncertainty of the synthesis of the standard uncertainty of each component is the synthesis uncertainty. The extension uncertainty gives the range of the measurement results where the measured value is located in a high confidence probability [8].

\subsection{Source of uncertainty}

The mathematical model of the measured parameter $y$ is multivariate function $y=f\left(x_{1}, x_{2}, x_{3}, \cdots x_{n}\right)$, and the value of $y$ depends on the uncertainty of other $n$ input quantity $x_{i}(i=1,2,3, \ldots n)$, that is

$$
Y=F\left(X_{1}, X_{2}, X_{3} \ldots X_{N}\right)
$$

From the best value $x_{i}(i=1,2,3, \ldots n)$ of each input $X_{i}$, the best value $y$ of $Y$ can be obtained, that is, the calculation result

$$
y=f\left(x_{1}, x_{2}, x_{3}, \cdots x_{n}\right) .
$$

Therefore, the uncertainty of the calculation result $y$ is derived from $x_{i}(i=1,2,3, \ldots n)$, that is, the uncertainty $u_{y}$ of $y$ depends on the uncertainty $u_{x i}$ of each input amount $x_{i}$.

The true value of each direct measured value ${ }^{x_{i}}$ is recorded as $x_{z}$, the uncertainty $u_{x i}$ of ${ }^{x_{i}}$ is expressed with absolute error $\Delta_{x i}$, and the uncertainty $u_{x i}$ of input
quantity ${ }^{x_{i}}$ is

$$
u_{x i}>\left|\Delta_{x i}\right|=\left|x_{i}-x_{z}\right|
$$

For the uncertainty of the measured parameter ${ }^{y}$, the calculation process is as follows.

\subsection{Calculate the standard uncertainty $u_{x i}$ of the input $x_{i}$}

The evaluation of standard uncertainty is divided into two categories which are evaluation of category A and evaluation of category B: category A is evaluated by statistical analysis of the observed columns, and calculate the standard uncertainty of the best estimation value, category $\mathrm{B}$ is evaluated by analyzing the distribution characteristics of the measurement columns to evaluate the standard deviation and the standard uncertainty, evaluation of category B plays an important role in the uncertainty evaluation. In view of the actual situation of the current road traffic accident scene investigation and the repeatability of the relevant data measurement, the velocity identification in the accident reconstruction model is suitable for the evaluation result of the evaluation method of category $\mathrm{B}[9,10]$.

By using the half width ${ }^{a_{i}}$ of the change interval of the input quantity $x_{i}$ to divide the inclusion factor ${ }^{k_{i}}$, the standard uncertainty $u_{x i}$ of the ${ }^{x_{i}}$ can be obtained 


$$
u_{x i}=\frac{a_{i}}{k_{i}}
$$

Inclusion factor $k$ is also known as a range factor. If the input quantity ${ }^{x_{i}}$ obeys the normal distribution, the inclusion factor is obtained by querying the normal distribution integral table; If the input quantity ${ }^{x_{i}}$ is uniformly distributed, the inclusion factor is $\sqrt{3}$ [8].

\subsection{Calculate the synthetic standard uncertainty $u_{y}$}

The standard uncertainty of the measured parameter $y$ caused by the standard uncertainty $u_{x i}$ from a single ${ }^{x_{i}}$ is:

$$
u_{y i}=\left|\frac{\partial y}{\partial x_{i}}\right| u_{x i}
$$

The relative change $\frac{\partial y}{\partial x_{i}}$ of the function $y$ corresponding to $u_{x i}$ is called the uncertainty transfer coefficient.

According to the theory of error synthesis, when each input quantity is independent, each input quantity will introduce a uncertainty factor, under their joint influence, the uncertainty of output $y$ shall be the synthesis of all uncertainty components, so the synthetic standard uncertainty ${ }^{u_{y}}$ of function $y$ is calculated from the following formula:

$$
u_{y}=\sqrt{\sum_{i=1}^{n} u_{y i}^{2}}=\sqrt{\sum_{i=1}^{n}\left(\frac{\partial y}{\partial x_{i}}\right)^{2} u_{x i}^{2}}
$$

\subsection{Calculate the extension uncertainty $U$}

The extension uncertainty is also called extended uncertainty, and the extension uncertainty $U$ can be obtained by multiplying the synthetic standard uncertainty $u_{y}$ with the inclusion factor $k$ multiplied by the function $y$.

$$
U=k u_{y}
$$

Using the extension uncertainty as the uncertainty of the measured parameter ${ }^{y}$, the function $y$ is expressed as

$$
Y=y \pm U
$$


This means that the best value given to the measured parameter $Y$ is ${ }^{y}$, which contain most of the value of $Y$ in the interval from $y-U$ to $y+U$, so the value range of the function value can be determined.

\section{The basic algorithm and uncertainty evaluation of vehicle velocity in vehicle impact accidents}

\subsection{The basic algorithm of vehicle velocity in vehicle scratch accidents}

The sides of the two cars scratch, or the contact surface of the impact head between the two cars is narrow, or the contact surfaces of the impact between the head of rear vehicle and the tail of the front vehicle is narrow, and the common velocity of the two vehicles is not existed in the compression process of the impact, so the impact pattern belongs to the scratch accident. In the process of vehicle' overtaking the center line or returning to the its lane, the scratch accident occurs more. The nature of the vehicle scratch accident is still a impact accident.

According to the law of conservation of energy, for an impact system composed of two impacting vehicles, the kinetic energy of a vehicle before impact in normal driving, and the energy transformation before and during the impact, are dissipated in the braking, sliding and deformation of the vehicle, etc. respectively. According to the braking or sliding distance after the vehicle impact, the deformation amount can be converted to the equivalent velocity component corresponding to a vehicle, and then synthesize the velocity of each part, and the driving velocity before the collision can be obtained [11].Therefore, the velocity of the impacting vehicle and the impacted vehicle before the scratch is equal to the velocity of the respective sliding after the impact of the two vehicles, plus their respective velocity changes in the course of the impact, that is

$$
v_{i 0}=v_{i}+v_{b i} \text {. }
$$

In the formula, $v_{i 0}$ is the instantaneous velocity of the vehicle before the scratch, $v_{i}$ is the instantaneous velocity of the vehicle after the scratch, $v_{b i}$ is the change of the velocity in the vehicle scratching process.

According to the law of conservation of energy, the kinetic energy of the vehicle is converted into friction work, and the instantaneous velocity $v_{i}$ can be calculated according to the vehicle sliding distance

$$
v_{i}=\sqrt{2 g \varphi_{i} s_{i}}
$$

In the formula, $g$ is the acceleration of gravity, $\varphi_{i}$ is the adhesion coefficient of the vehicle, $S_{i}$ is the sliding distance after the vehicle scratch.

According to the law of conservation of energy, the velocity variation of the vehicle before and after the scratch is $v_{b}$, the corresponding equivalent velocity of which can be calculated by the empirical formula obtained from the test according to the deformation $D$ of the part of the impact 


$$
v_{b i}=h D_{i}
$$

In the formula, $D_{i}$ is the deformation of the vehicle impact parts, $h$ is the ratio constant between the velocity and deformation, and is generally obtained by empirical formula.

The formula (10) and the formula (11) are brought into the formula (9) to obtain the instantaneous driving velocity $v_{i 0}$ of the vehicle before the scratch which is:

$$
v_{i 0}=v_{i}+v_{b i}=\sqrt{2 g \varphi_{i} s_{i}}+h D_{i}
$$

\subsection{Uncertainty evaluation of instantaneous velocity of vehicle before scratch}

The parameters of the uncertainty associated with the instantaneous velocity of the vehicle are removed, excluding the gravity acceleration $g$, including $\varphi_{i}, S_{i}$ and $D_{i}$, where the second order uncertainty is introduced by $\varphi_{i}$ and $S_{i}$. The relationship between the velocity of the vehicle and its uncertainty can be expressed as

$$
v_{i 0}=f_{i}\left(\varphi_{i}, s_{i}, D_{i}\right) \text {. }
$$

When evaluating the uncertainty of the instantaneous velocity before the vehicle scratch, the standard uncertainty $u_{x i}$ of each input quantity $x_{i}\left(\varphi_{i}, s_{i}, D_{i}\right)$ shall be calculated first

$$
\begin{aligned}
& u_{\varphi i}=\frac{a_{\varphi i}}{k_{\varphi i}} . \\
& u_{s i}=\frac{a_{s i}}{k_{s i}} . \\
& u_{D i}=\frac{a_{D i}}{k_{D i}} .
\end{aligned}
$$

Secondly, the standard uncertainty component $u_{v i 0 . x i}$ of each input quantity $x_{i}\left(\varphi_{i}, s_{i}, D_{i}\right)$ is determined

$$
\begin{aligned}
& u_{v i 0 . \varphi i}=\left|\frac{\partial v_{i 0}}{\partial \varphi_{i}}\right| u_{\varphi i}=\sqrt{\frac{g s_{i}}{2 \varphi_{i}}} \cdot u_{\varphi i} . \\
& u_{v i 0 . s i}=\left|\frac{\partial v_{i 0}}{\partial s_{i}}\right| u_{s i}=\sqrt{\frac{g \varphi_{i}}{2 s_{i}}} \cdot u_{s i} .
\end{aligned}
$$




$$
u_{v i 0 . D i}=\left|\frac{\partial v_{i 0}}{\partial D_{i}}\right| u_{D i}=h \cdot u_{D i}
$$

Evaluate the synthetic standard uncertainty $u_{v i 0}$ of the velocity of the identified vehicle $v_{i 0}$ before scratch and impact

$$
u_{v i 0}=\sqrt{\sum_{i=1}^{n} u_{v i 0 . x i}^{2}}=\sqrt{u_{v i 0 . \varphi i}^{2}+u_{v i 0 . s i}^{2}+u_{v i 0 . D i}^{2}}
$$

By multiplying the synthetic standard uncertainty $u_{v i 0}$ of the function $v_{i 0}$ with the inclusion factor $k$, the extension uncertainty $U_{v i 0}$ of the function $v_{i 0}$ can be obtained.

$$
U_{v i 0}=k u_{v i 0}
$$

Using the extension uncertainty as the uncertainty of the measured parameter $v_{i 0}$, the measured driving velocity $v_{i 0}$ before the vehicle impact is expressed as

$$
V_{i 0}=v_{i 0} \pm U_{v i 0}
$$

This means that the optimum value of the measured parameter is $v_{i 0}$, which can contain most of the $V_{i 0}$ in the range of $v_{i 0}-U_{v i 0}$ to $v_{i 0}+U_{v i 0}$, and can determine the range of driving velocity before the vehicle scratch.

\section{Case analysis of actual accidents}

The passenger car is driving from west to east, its left front wheels cross the yellow line in the middle of the road which is in the early overtaking stage, the truck is driving from east to west, its left front wheels press the yellow line in the middle of the road which is in the late overtaking stage, and is returning to its own lane, and the impact occur at the left front corner when the two vehicles are overtaking across the middle line. The key content of accident identification is the velocity before the impact of the passenger car.

The passenger car in the accident is driving opposite to the truck, there is no common velocity in the impact, the impact form is the reverse scratch, and the driving velocity $v_{10}$ before the impact of the passenger car shall be equal to the sliding velocity after its impact $v_{10}$ plus the change of its velocity in the impact $v_{b 1}$.

$$
v_{10}=v_{1}+v_{b 1} .
$$




\subsection{The instantaneous driving velocity $v_{10}$ of the passenger car before scratch}

According to the accident scene picture, after impact, the distance between the passenger car on the road is $s_{1}=34.1 \pm 0.10 \mathrm{~m}$, the angle between the longitudinal axis and the longitudinal axis is $53.5^{\circ}$, considering that the passenger car is not braking and has irregular rotation and sideslip, the comprehensive estimate of $[12,13,14]$ has its adhesion coefficient $\varphi_{1}=0.26 \pm 0.02$, and the average deformation is $D_{1}=0.32 \pm 0.02 \mathrm{~m}$ if the head of the bus is calculated on a double curved surface, and the total mass of the truck is more than $17000 \mathrm{~kg}$, so the ratio constant between the velocity of the passenger car and deformation is $h=67$ [15], according to the law of conservation of energy, the instantaneous velocity $v_{10}$ of the passenger car before the scratch is obtained

$$
v_{10}=v_{1}+v_{b i}=\sqrt{2 g \varphi_{1} s_{1}}+h D_{1}=68.863 \pm 3.236 \mathrm{~km} / \mathrm{h} .
$$

\subsection{Evaluation of uncertainty of driving velocity $v_{10}$ of the passenger car before scratch}

The vehicle velocity identification of road traffic accidents is evaluated by the B-type evaluation method. According to the uncertainty theory, take the value of the inclusion factor corresponding to the confidence probability 0.95 as $k=2$, calculate according to formula (14) (22), and the standard uncertainties of the parameters $\varphi_{1}, s_{1}$ and $D_{1}$, which are related to the driving velocity $v_{10}$ before the passenger car scratch with the uncertainty, are $u_{\varphi 1}=0.01, u_{s 1}=0.05 \mathrm{~m}$ and $u_{D 1}=0.01 \mathrm{~m}$, respectively, and the standard uncertainty components are $u_{v 10 . \varphi 1}=0.913, u_{v 10 . s 1}=0.0348 \mathrm{~m}, u_{v 10 . D 1}=0.67 \mathrm{~m}$, where the uncertainty coefficient of the adhesion coefficient $\varphi_{1}$ and the deformation amount $D_{1}$ of the collision site is large, which has a significant effect on the calculation results of the velocity before the impact, in contrast, the uncertainty of the sliding distance $S_{1}$ after the vehicle scratch is very small. After comprehensive calculation, the synthetic standard uncertainty is $u_{v 10}=1.133 \mathrm{~km} / \mathrm{h}$ and the extension uncertainty is $U_{v 10}=2.266 \mathrm{~km} / \mathrm{h}$, then what can be obtained is that the interval of the velocity value of the passenger car before the scratch is

$$
v_{10}=68.863 \pm 2.266 \mathrm{~km} / \mathrm{h}=66.597 \sim 71.129 \mathrm{~km} / \mathrm{h}
$$

The conclusion of this case is that the velocity of the passenger car is $69 \mathrm{~km} / \mathrm{h}$ before the impact. 


\section{Conclusion}

Without considering the error of geometric parameters in the field, in this paper, the uncertainty of the parameters such as the adhesion coefficient of vehicle and road surface, the sliding distance after scratch and the deformation of the vehicle impacting part are analyzed in the road traffic accident under the condition of scratch form of vehicles. The uncertainty of the degree of deformation introduced by the adhesion coefficient and the impact site has significant influence on the calculation results of the vehicle velocity before impact, and the uncertainty calculation method for the velocity identification is obtained, so as to determine the range of the driving velocity before the vehicle impact.

\section{References}

1. Yuan Q., Chen H.Y., Li Y.B.: Using Injury Severity Data to Determine the Impact Speed of Traffic Accidents between Vehicle and Vulnerable Road User. J. Chinese Journal of Forensic Sciences. V86 (3), 32-39 (2016)

2. Chen T., Wei L., Gong B., Zhou W.X.: Parameter Sensitivity Analysis of Reconstruction System for Vehicle-Vehicle Collision Accidents. J. Automotive Engineering. 34(9), 771-776 (2012)

3. Zhang J., Zhang X., Li J., Ni X. D., Gao J. G.: Sensitivity Analysis on the Selected Parameters in Typical Vehicle Collision Models. J. Automotive Engineering. 34(10), 905-908 (2012)

4. $\mathrm{Hu}$ N., Lu G.Q., Li T., Li Y.B.: Calibration Precision Experiments Analysis of Photogrammetry in Traffic Accident Scene Based on Separately-calibrate Method. J. Automotive Engineering. 30(7), 557-560 (2008)

5. Liu Z.Q., Wang P., Zhang J.H., Wang Y., Gong B.: Review of Traffic Accident Reproduction Technology and Its Developing Trend. J. China Safety Science Joumal. 17(4), 16-20 (2007)

6. Yuan Q., Zhang G. Q., Guo R., Li Y.B.: Research Evolution on the Theory Methods of Vehicle-speed Analysis of Traffic Accidents. J. Journal of Transportation Engineering and Information. 7(2), 21-27 (2009)

7. Yuan Q., Li Y.B.: Influence of Parametrical Uncertainties on Traffic Accident Reappearance of Vehicle Side Impact. J. Transactions of the Chinese Society for Agricultural Machinery. 36(5), 16-19 (2005)

8. Fei Y.T., Error theory and data processing. M. China Machine Press. (2015)

9. Tan D. R., Yin P.: Speed Estimation for Vehicle-Bicycle Side Impact Accident Based on the Uncertainty Theory. J. Automotive Engineering. 32(3), 220-222 (2010)

10. Deng H.Y., Mo Y.Z., Jiang S.X., Han Z.G.: Application of the Uncertainty Evaluation Algorithm to Automobile Accident Recurrence. J. Journal of Central South University of Forestry \& Technology. 26(4), 96-100 (2006)

11. Yuan Q., Li Y.B.: Uncertainty Evaluation on the Energy Method of Vehicle Traffic Accident Reconstruction. J. China Journal of Highway and Transport. 15 (1), 110-112 (2002)

12. Li C. C., Liu X. M., Rong J.: Experimental Study on Effect of Road Condition on Pavement Friction Coefficient. J. Journal of Highway and Transportation Research and Development. 27(12), 27-32 (2010)

13. Sui B.: Research on the Traffic Accidents Vehicle-Speed Reproduction Methods. D. Harbin Institute of Technology. (2012)

14. Yu Z. P., Zuo J. L., Zhang L.J.: A Summary on the Development Status Quo of TireRoad Friction Coefficient Estimation Techniques. J. Automotive Engineering. 28 (6), 546-549 (2006) 
15. General Administration of Quality Supervision, Inspection and Quarantine of P. R. C., Standardization Administration of P. R. C.: Identification for the Speed of Vehicle Involved in Road Traffic Accidents. S. China Standard Press. GA/T 33195-2016 (2016) 